\title{
AS VIAS BILÍFERAS DOS TATUS (Dasypus novemcinctus e Euphractus sexcinctus)
}

\author{
TIIE BII.IARY TREE IN ARMADIILOS (Dasypus novemcinctus and Euphractus sexcinctus)
}

\author{
Wilson Machado de S()U7.A'; Maria Angélica MI(GI.INO²; Irvenia Luiza de Santis PRADA³ I iberato João Afonso DII)IO(
}

\section{RESUMO}

()s autores esquematizam as vias bilíferas de 2 espécies de latus (Dasypus novemcinctus c Euphractus sexcinctus) oriundos do Pantanal Matogrossense. Brasil. A dissecação foi efetuada após o preenchimento do sistema excretor do fígado com látex Neoprene 6.50 corado e da lixação das peças em solução aquosa de lormol a 10\%. O ductus choledocus. em ambas as espécies, provém da reuniäo do ductus cysticus com o ductus hepaticus. Este, por sua vez, resulta da convergência do ramus principalis dexter e do ramus principalis sinister. Na formação dos ramus principalis dexter e sinister participam vários ramos oriundos dos lobos hepáticos e seus processos.

UNITERMOS: Ductos biliares; Animais silvestres; Fígado

\section{INTRODUÇÃO}

As vias de drenagem biliar foram objeto de diversos trabalhos, em várias espécies silvestres, a saber, girafa (PRADA et al. ${ }^{7}$. 1975): macaco prego (BORELLI el al. ! 1975); gambá (SILVA et al. "12, 1983); zebra (D'ERRICO el al. ${ }^{2}$, 1983); veado (MIGLINO et al.*); capivara (MIGLINO el al.5, 1986); tamanduá bandeira (SOUZA ct al. $\left.{ }^{13}, 1992\right)$; leão (PRADA et al." 1988); anta (MIGLINO et al. ", 1982); cateto (MIGI.INO et al. $\left.{ }^{6}, 1986\right)$ : ratão do banhado (PRAI)A cl al. ${ }^{8}, 1981$ ). Nesta publicação, relataremos o trabalho, o comportamento das vias bilíferas em duas espécies de latus, o Dasypus novemcinctus e o Euphractus sexcinctus e sua relação com os lobos do fígado. Os resultados obtidos serāo comparados com os do tamanduá bandeira, que lambém pertence aos Edentata (Xenarthra).

\section{MATERIAI, E MÉTODO}

Utilizamos 8 tatus. sendo 4 ( 3 machos c 1 fêmea) talus galinha (Dasypus novemcinctus) e 4 tatus peba (Euphractus sexcinctus), machos, oriundos de diversals regiōes do Pantanal Millengrossense. Brasil.

Cada preparação comprecondia. depois de comveniente io dução, lígado e parte do trato intestinal correspondente a localização da papila duodenal maior. Esta porção da alça intestinal foi aberta. ao longo da sua borda livre, esvaziando-se, logo depois, o maximo possível, as vias biliferas. mediante suaves massagens.

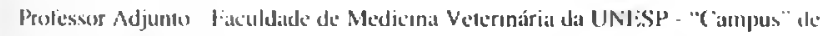
Arib̧illuba

- Professor Aswidielo Faculdade de Medicina Veterinaria e Zootecnia da USP

- Professor Tiuular - Faculdade de Modicina Veterinairia e /asetecnia da USP

4- Professor Visitante - Escola Patulista de Medicina
Imediatamente após, o ducto colédoco foi canulado, através da papila, injetando-se, as vias bilíferas, com Neoprene látex 450, corado. Depois estes órgãos foram fixados cm solução aquosa de formol a $10 \%$, para posterior dissecção e esquematização destas vias excretoras, pela face visceral.

Adotamos a nomenclatura sugerida por JABLAN-PANTIC ${ }^{3}$ (1963). Convencionamos ainda, dividir os lígados, conforme sugerem SCHUMMER; NICKEL ${ }^{10}$ (1979), para os animais de um modo geral, em três partes, esquerda, intermédia e dircita, valendo-nos de duas linhas imaginárias traçadas sobre a face visceral dos órgãos, em correspondência a alguns acidentes anatômicos. Deste modo, uma delas se sobrepōe ao eixo maior da fossa da vesícula biliar, atingindo dorsalmente a impressāo da veia cava caudal, enquanto a outra alcança at incisura do ligamento redondo e a impressão esofáigica.

Servindo-nos das incisuras que o fígado apresenta, identificamos: o lobus dexter lateralis e o lobus dexter medialis: o lobus sinister lateralis e o lobus sinister medialis: na porção inlraportal, o lobus quadratus; o lobus caudatus, integrado ’’’ processus caudatus, pela pars supraportalis e, no tatu ":alinha, também, pelo processus papillaris.

\section{RESULTADOS}

I:In lodas as preparaçōes, o ductus choledocus provém da união do ductus hepaticus e ductus cysticus, independentemente da espécic considerada (Fig.1 e 2). Por sua vez, o ductus hepaticus origina-se constantemente a partir da con-

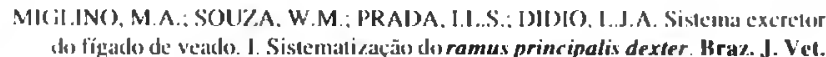
Res. anim. Sci.,Sia Paulo. (No prelo) 


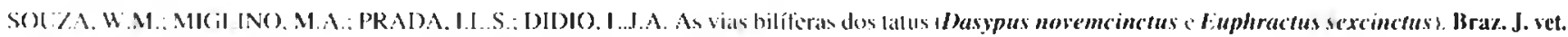

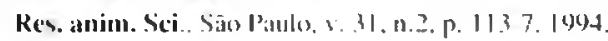

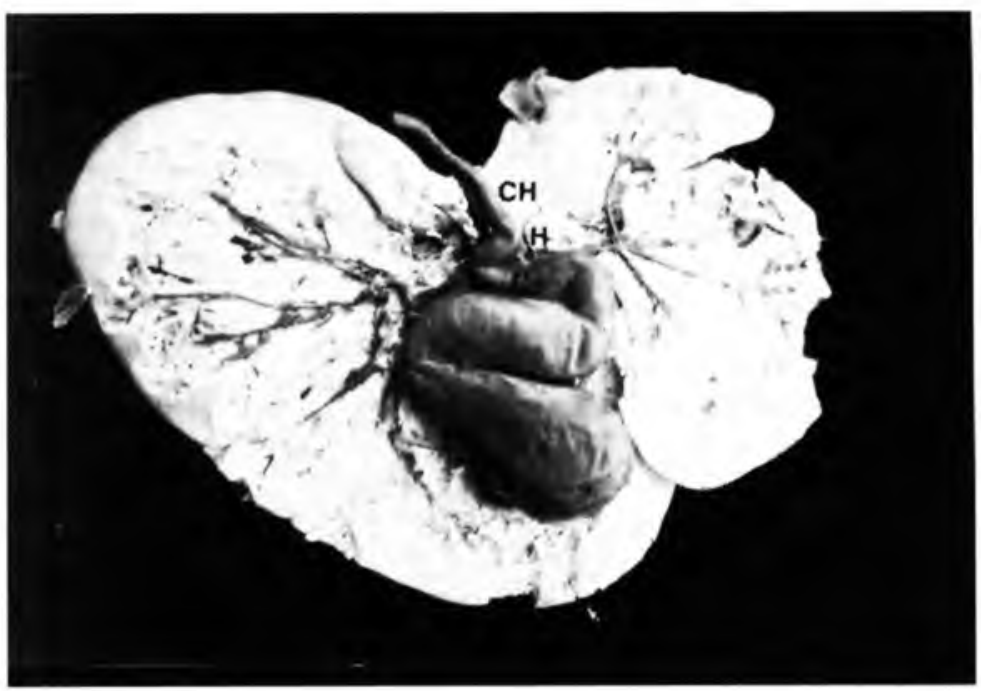

Fl(il Ri 1

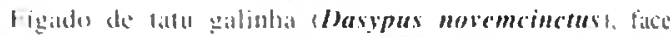
lisceral. () ducrus choledocus ic Hi proscill dis uniảo do ductus heparicus (H) cum o ductus cysicus (C); ('ste. por

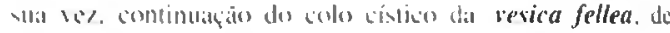

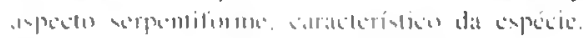

FIGLRI 2

Fífidde de lallu petha teuphractus sexcinctus ) lace visceral O ramus principalis dexter (d) aprescula cuunos tomponente iniciain. oramus processi caudati (c) mais o ramus lateratis lobi dexiri \&ldh, () ramus principalis sinister (s). original so da reunuo do ramus lateralis tohi sinistri (ls) e ramus medialis lohi sinistri Imst mais ot romus lobi quadrati (4).

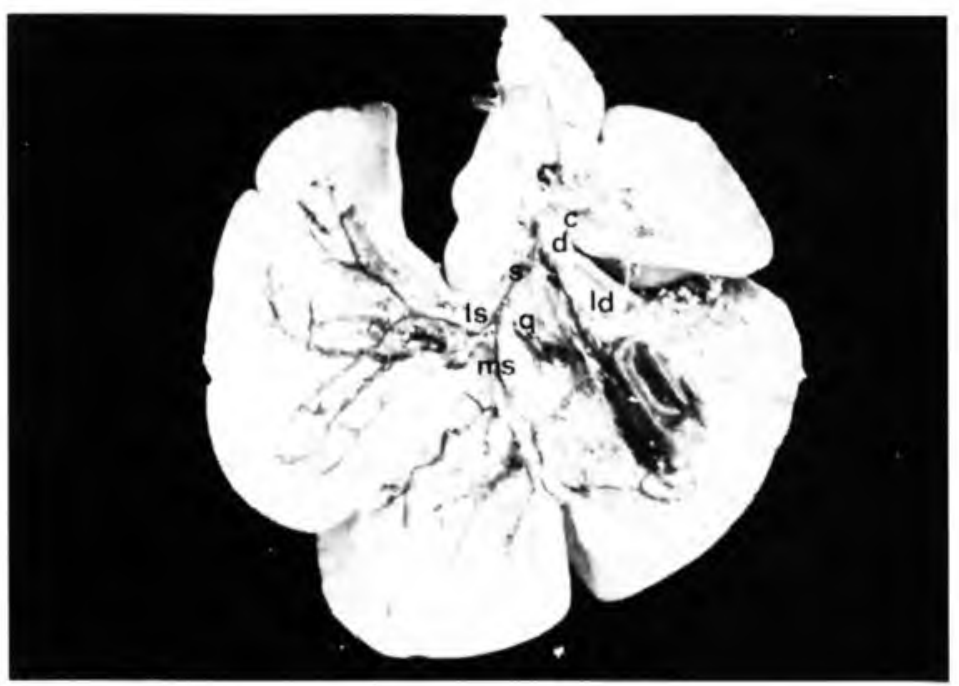

liluência do ramus principalis dexter c do ramus principalis sinister. Lanto no Dasypus novemcinctus quanto no Euphractus sexcinctus. sem apresentatr tributários (Fig. I e 2)

O ductus cysticus une-se ao ductus hepaticus. para originar o ductus choledocus. sendo visto $\mathrm{cm}$ todas as peças do tatu galinha. sem atluentes. o mesmo ocorrendo em 2 das 4 glândulas do tatu peba; nas outras 2 peças deste grupo. o aludido coletor recebe a meio caminho o ramus medialis lobi dextri (Fig.2).

A vesica fellea, no tatu pebal, apresenta-se esférica c bastante inclusa no parênquima hepático a ponto de sua fossa alcançar a face diaframática do fígado. No tatu galinha, esse reservatório mostrou-se elipsóide, de disposição scrpentiforme c relacionada à discreta lossa do parênquima do lígado (Fig. I).

O ramus principalis dexter (Jïg. I), no lasypus, provem da uniāo do ramus processi caudati c do ramus lateralis lobi dextri. sem coletores e lormando tronco, ato qual vern ter 2 afluentes independentes do lobus dexter (território lateral). em 2 fígados. Nos outros 2 orgãos. o citado tronco encontrase desprovido de eferentes. A este tronco entretanto. vem ter. ainda, tributátio do lobus caudatus (pars supraportalis) e 0 ramus medialis lobi dextri. em todas as peças do tatu galinha.

Nos lígados de Euphractus sexcinctus, o ramus principalis dexter resulta apenas da confluencia do ramus processi caudati mais ramus lateralis lobi dextri, em 2 órgãos. e por estes dois coletores. sempre lormando tronco, ao qual vem a ter o ramus medialis lobi dextri nas outras 2 glândulas. () ramus processi caudati recebe tributário independente. oriundo do lobus dexter (território latcral), em 1 destes 4 fígados.

O ramus principalis sinister. em todas as peças do gênero Dasypus e. em três das quatro glândulas do Euphractus, 
provém da contluência do ramus lateralis lobi sinistri mais o ramus medialis lobi sinistri. o qual recebe. em 3 das 4 peças do taltu gallinhat. tributírio do lobus sinister (território latcral). Vo outro fígado de tatu peba, o ramus medialis lobi sinistri aflui ao ramus lobi quadrati (Fig.2), compondo deste modo) tronco comum desprovido de afluentes.

Achat-se. igualmente, sem tributários, em ambas as espécies. a ducto primeiramente descrito, que recebe a seguir, o ramus lobi quadrati em 7 das 8 peças, compondo assim, o ramus principalis sinister em 3 fígados do latu peba. No outro casos. o ramas lateralis lobi sinistri unc-se ao tronco formado pelo ramus medialis lobi sinistri mais o ramus lobi quadrati. palra compor a referida via.

Com referência ao tatu galinha, o duco constituído pela convergência do ramus lateralis lobi sinistri mais o ramus medialis lobi sinistri c ramus lobi quadrati. recebe, a yeguir. o ramus processi papillaris, sem alluentes, para, desla maneira. individualizar oramus principalis sinister, igualmentc sem iributários.

\section{DISCUSS̃̃o}

Vários raballhos foram efetuados em mamíteros silvestres. com ohjetivo de siscematizar as vials biliferas intrat é cexlla hepáticas. latis sejam. makaco prego (B3()RELL.I et al. '. 1975): girata (PRAI)A et al. . 1975): ratão do banhado (PRAI) A (1)

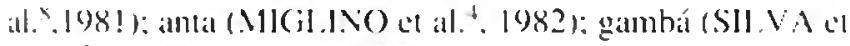
al. ${ }^{11.12}$. 1983): acbra (1) ERRICO et al. ${ }^{2}$ 198.3): calc(u

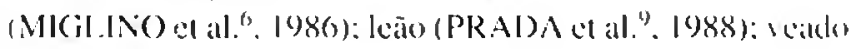
(NIICIINO et al. "): lamanduá bandeira (SOUKA et al.". 1992).

Os resultados desses trabalhos permitem. com as devidas restriçies , um cotejo com os ora vistos, para o comportamento destas estruturas no tattu. despertando particular interesse, o contronto destes dados. com os observidos no tamanduá bandeirat. por se tralar de espécie também pertencente ao grupo Edentata. De fato. mortologicamente, o ligado do tamanduá mostra seus contornos semelhantes ao do latu peba, inclusive o formato ligciramente esférico da vesica fellea. embora em menores proporçōes. lintretanto, não se nota no Myrmecophaga. uma fossa para a vesícula biliar tão ampla. que chegue a alcançar a face diafragmática do fígado, como ocorre no tatu peba. Já relativamente ao gênero Dasypus, a vesícula biliar mostra um formato de clipsóide alongado, de aspecto serpentiforme. Aliás, formato semelhante, porém nāo tão acentuado. pode-se observar no fígado do macaco prego (BORELLI et al.' .1975).

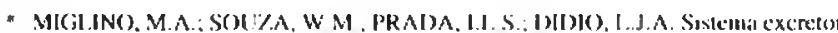
do figadu de veidh. I. Sistematı/ẫu do ramu. principalis dexter. B raz. J. Vet. Res. anim. Sci..Sàu Paulu. (Nôn prelen)
}

O ductus hepaticus no latu é proveniente da união do ramus principalis dexter com o ramus principalis sinister e independentemente do gênero, une-se ao ductus cysticus pari compor oductus choledocus; este comportamento também é visto constantemente. no tamanduá bandeira (SOLZA el al. 1:. 1992), em grande proporção (75\%) no macalco prego

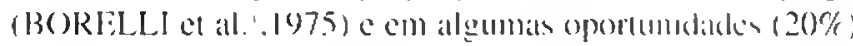

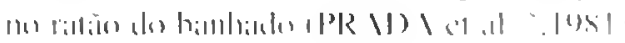

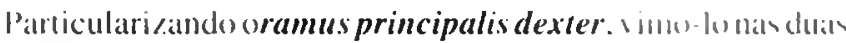
espécies de tatus ora estudadas, composto inicialmente pela convergência doramus processi caudati mais o ramus lateralis lobi dextri: formando tronco comum. a) qual vem ter, em todas as peças do tatu galinha, e cm duas no tatu peba. oramus medialis lobi dextri; nas outras duas glândulas estc último colctor vai ter an ductus cysticus, não participando portanto da composição do ramus principalis dexter. Esta formação. alora este último aspecto. se tomada genéricamente. não di fere dacuela observada no tamanduá bandeira. que cxibe. constituir a referida via. os mencionados componente nominados, salvo cm apenas uma oportunidade. onde orama. processi caudati não participa do aludido ducto. Relativa mente ao ramus principalis sinister, verificamos a participit rem constantemente de sua formação, o ramus lateralis lobi sinistri. oramus medialis lobi sinistri. oramus lobi quadrati L' no latu galinha o ramus processi papillaris, nio observado mo lígado do latu pebal. Os três componentes comuns a andbas espécies de tatus, configuram apenas um único arranjo no Dasypus. ou seja, o primeiro mais o segundo, compondo tron(o). que acolhe o terceiro. disposição nào observada em apenals uma preparação no tatu peba. quando oramus lobi quadrati unc-se as ramus medialis lobi dextri constiluindo tronco convergente ao ramus lateralis lobi sinistri. Resultado idênlico a cste último aspecto foi assinalado no tamanduá bandeira cm igual proporção, animal onde pode-se verilicar também. a participação eventual (25\%). na formação do ramus principalis sinister. do ramus processi caudati. evento não) deteclado, quer no Dasypus quer no Euphractus. O ramus processi papillaris. não identiticado no tatu peba co no tamanduá bandeira. mas visto, no tatu galinha, constantemente a fazer do ramus principalis sinister, ja foi registrado $\mathrm{cm}$ outras espécies silvestres, ou seja, macaco prego (BORLiLI.I el al.'. 1975), ratão do banhado (PRADA el al. ${ }^{8} .1981$ ) eleão(PRAD)A et al. ", 1988). Aliás. os fígados destes espécimes, exceção feita ao último deles, apresentam morfologia hastante semethante ao dos gêneros ora estudados, inclusive relativamente às incisuras de separação dos lobos hepáticos, mais ou menos profundas na dependência da espécie considerada. 
SOUZ.A. W.M.: MIGL.INO, M.A.: PR.ADA. I.L.S.; DIDIO. L.J.A. As vias bilíferas dos latus (I)asypus novemcinctus e Euphractus sexcinctus"). Braz. J. vet. Res. anim. Sci., São Paulo, v. 31, n.2, p. 113-7, 1994.

\section{CONCLUSÕES}

01. O ductus choledocus no tatu galinha e Dasypus novemcinctus e no tatu peba Euphractus sexcinctus estudadas provém da reunião do ductus cysticus com o ductus hepaticus

02. O ductus hepaticus, constantemente sem afluentes, resulta da convergencia do ramus principalis dexter e ramus principalis sinister, lanto na espécie Dasypus novemcinctus como no Euphractus sexcinctus.

03. São componentes do ramus principalis dexter, o ramus processi caudati, o ramus lateralis lobi dextri e o ramus medialis lobi dextri, na espécic Dasypus novemcinctus e em 50\% das peças da espécic Euphractus sexcinctus. Nos casos restantes desta espécic, o último coletor unese ao ductus cysticus.
04. O ramus principalis sinister, nas duas espéciess estudadas, apresenta scmpre na sua constituição, o ramus lateralis lobi sinistri. o ramus medialis lobi sinistri co ramus lobi quadrati. No Dasypus, além destes. fà parte do referido ducto. o ramus processi papillaris

05. Tributários foram identificados para o ramus processi caudati, oriundos do lobus dexter (território lateral). I ve\% no Euphractus. Com esta mesma origem. 2 tributários. em 2 casos. foram notadas afluindo para o tronco formado pela união do ramus processi caudati c do ramus lateralis lobi dextri. no Dasypus; c procedentes do lobus caudatus (pars supraportalis). para o mesmo tronco. foram vistos em todas as peças de ambos os gêneros.

\section{SUMMARY}

The biliary tree of armadillos (Dasypus novemcinctus e Euphractus sexcinctus). from the Pantanal Matogrossense. Brazil. was studied after latex injection. formaldehyde fixation and dissection. The ductus choledocus. in hoth species, results from the junction of the ductus hepaticus and the ductus cysticus. The ductus hepaticus is made up by the convergence of the ramus principalis dexter and the ramus principalis sinister. in turn formed by branches of hepatic lobes.

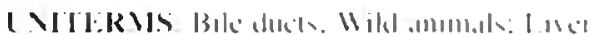

\section{REFERÊNCIAS BIBLIOGRÁFICAS}

01-BORFLLI, V.; PRADA, I.L.S.; FERNANDES FII,HO, A.; ANDRETTO. R. Contribuiçāo ao estudo do sistema excretor do fígado em animais silvestres. II. Vias bilíferas do macaco prego (Cehus sp.). Rev. Fac. Med. Vet. Zootec. Iniv. S. Paulo. v.12, p.67-9)4. 1975 .

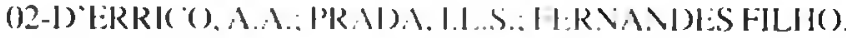
A. Contribuição an estudo dats vias bilíferas na ecbra (Equus zebra L.1758). In : SEMANA DE MEDICINA VETERINÁRIA. 2., São Paulo, 1983. Anais. p.7.

03-JABI.AN-PANTIC, O. Characteristics and comparative rations of intrahepatic bile duct in domestic animals. Actd. Vet.. Beograd. v. 13, p.3-4, 1963.

04-MIGLINO, M.A.; PRADA. I.L.S.: D'ERRICO, A.A.; SOUZA, W.M. Contribuição ao estudo do sistema excretor do fígado em animais silvestres. V. Vias bilíferas da anta (Tapirus americanus). In: SEMANA DE MEDICINA VETERINÁRIA, 1., São Paulo, 1982. Anais. p. 38.
05-MIGLINO. M.A.: PRADA, I.L.S.; SOUZA. W.M.: D’ERRICO. A.A. Contribuição ao estudo do sistema excretor do fígado em animais siluestres. V. Vias bilileras da capivara. (Hydrochoerus hydrochoeris). Rev, Fac. Med. Vet. Zootec. Univ. S. Paulo, v. 23. D. II.5.32. 1980

()6-MIGLINO, M.A.; SOU\%A, W.M.; NASCIMI:NTO, A.A. ARANTES, J.G.: CARVALHAL. R. Contribuição at) estudo do sistema excretor do fígado cm animais silvestres. Vias bilíferas do cateto (Tayassu tayassu). In: CONGRI:SSO BRASIIIIRO DE MEDICINA VFTIERIVÁRIA. 20). Cuiabí. 1980. Inais. p.342.

(17-PR.SDA. I.L..S.: BOOREL.L.I. V.: PEDUTII NETO, J. Contribuição ao estudo do sistema excretor do fígado em animais silvestres. I. Vias bilíferas da girafa (Giraffa camelopardalis). Rev. Fac. Med. Vet. Zootec. Univ. S. Paulo, v.12, p.45-52, 1975.

08-PRADA. I.L.S.: D'ERRICO, A.A.; BORLI.I.I. V.: SOGORB, S.F. Contribuição ao estudo do sistema excretor do fígado $\mathrm{em}$ animais silvestres. Ill. Vias 
bilileras do lallion do bambado (.Myocastor coypus s. In: CONGRESSO BRASILEIRO DE ANATOMIA. 13.; CONGRESSO LUSO-BRASILEIRO DE. ANATOMIA, 4.. Bclo Horizonte, 198. Anais. p. 185.

(19-PRADA. I.L.S.: D'ERRICO, A.A.; SANTOS, R.M.B.: SOUSA, M.R.Q. Sistema excretor do fígado em animais silvestres. VII. Vias bilíferas do leão (Pantera leo ). In: CONGRESSO BRASILEIRO DE ANATOM11. 15.: CON(IRI:SSO 1.L'SO-BRASILEIRO DF ANATOMI: 5. 5. Brisília, 1988. Anais. p.1+2.

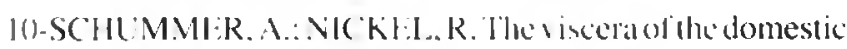
mammals. In: VICKLL. R: SCHUMMER, A.; SEIFERI.E, 1:; SACK. W.O. Textbook of the anatomy of domestic animals. Berlin. Paul Parey, 1979

11-SILVA, L.E.B.; FERREIRA, N.; SOUZA, A.; FAGUNDES, G.M. Contribuição ao estudo das vias bilíferas intra e extra-hepáticas em gambás (Didelphis aurita). Sistemalização do ramus principalis dexter. In: SEMANA DE MEDICINA VETERINÁRIA, 2., São Paulo, 1983. Anais. p.42.
I 2-SII.VA, L.E.B.; FERREIRA. N.; SOUZA, A.; FAGUNDES, G.M. Contribuição ao estudo das vias bilíferas intra e extra-hepáticas em gambás (Didelphis aurita). Sistematização do ramus principalis sinister. In: SEMANA DE MEDICINA VETERINÁRIA, 2., São Paulo, 1983. Anais. p.43.

I.SOUZA, W.M.; MIGLINO, M.A.; PRADA. I.L.S.; BORELLI, V.: ARANTES, I.G.; SOUZA, N.T.M. The excretory system of the liver in wild animals. VI. Biliary ducts of the great anteater (Myrmecophaga tridactyla). Braz. J. vet. Res. anim. Sci., São Paulo, v.29, p.353-7, 1992. Suplemento.

Recebido para publicação em 05/04/93 Aprovado para publicação em 19/10/93 